\title{
Determinación de la ruta crítica para la síntesis Química de hidroxiapatita nanométrica
}

\section{Determination of critical route for nanometric hydroxyapatite Chemical synthesis}

\author{
SOTO-TAPIZ, Mónica†*, GARCÍA-GALLEGOS, Juan y MARTÍNEZ-ALVARADO, Luis
}

Universidad Autónoma de Baja California, Facultad de Ingeniería Mexicali

ID 1 ${ }^{\mathrm{er}}$ Autor: Mónica, Soto-Tapiz / ORC ID: 0000-0002-1272-049X

ID $1^{\text {er }}$ Coautor: Juan, García-Gallegos / ORC ID: 0000-0002-8532-3241

ID $2^{\text {do }}$ Coautor: Luis, Martínez-Alvarado / ORC ID: 0000-0002-2193-0456

DOI: $10.35429 / J C P E .2019 .20 .6 .28 .32$

Recibido 09 Junio, 2019; Aceptado 28 Septiembre, 2019

\section{Resumen}

Actualmente, el área de atención a la salud demanda materiales con adecuadas propiedades mecánicas, biocompatibles $\mathrm{y}$, para ciertas aplicaciones, de alta bioactividad al estar en contacto con ambientes biológicos; así mismo, que respondan al incremento de la expectativa de vida y el acrecentamiento de enfermedades crónico degenerativas de la población. Por ejemplo, los adelantos en implantología han facultado a los investigadores para conocer las reacciones que ocurren entre los tejidos y los diversos implantes. En este sentido, las biocerámicas son un tipo de material bioactivo, diseñadas específicamente para la manufactura de implantes quirúrgicos, prótesis y órganos artificiales; así como para cumplir una determinada función fisiológica en el cuerpo humano dado a que poseen una buena biocompatibilidad y osteointegración, debido a la similitud que comparten con el componente mineral del hueso, por lo que sus expectativas de aplicación son muy amplias. Ante las consideraciones mencionadas, se diseñó la ruta crítica a seguir para realizar la síntesis de polvos de HA nanométrica mediante el método químico de combustión. Lo anterior aportará información sobre los reactivos precursores, el agente combustionante y con ello; las temperaturas y tiempos de reacción que darán como resultado la obtención de un producto de calidad que cuente con las características estequiométricas preestablecidas.

Biocompatible, Hidroxiapatita, Bioactivo

\begin{abstract}
Currently, the health care area demands materials with appropriate mechanical and biocompatible properties, and for some special applications, high bioactivity is important since these materials are in contact with biological environments; also, it's important that they respond to the population's increment in life expectancy and the increase in chronic degenerative diseases. For example, advances in implantology have enabled researchers to know the reactions that occur between tissues and diverse implants. In that sense, bioceramics are a type of bioactive material, specifically designed for the manufacture of surgical implants, prostheses and artificial organs, as well as to fulfill a certain physiological function in the human body due to their good biocompatibility, osseointegration and the similarity they share with the bones mineral components, so their application expectations are many. Based on the above considerations, a critical synthesis route was designed to synthesize nanometric HA powders by chemical-combustion method. This will provide information about the precursors reagents as the combustion agent, and, thereby; the temperatures and reaction times that will result in obtaining a quality product that has the pre-established stoichiometric characteristics.
\end{abstract}

Biocompatible, Hydroxyapatite, Bioactive

Citación: SOTO-TAPIZ, Mónica, GARCÍA-GALLEGOS, Juan y MARTÍNEZ-ALVARADO, Luis. Determinación de la ruta crítica para la síntesis Química de hidroxiapatita nanométrica. Revista de Energía Química y Física. 2019. 6-20: 28-32

\footnotetext{
* Correspondencia al Autor (Correo electrónico: soto.monica@uabc.edu.mx)

$\dagger$ Investigador contribuyendo como primer Autor
} 


\section{Introducción}

El desarrollo de la sociedad del conocimiento y el impacto que los insumos derivados de dicho proceso evolutivo pero sensible a las necesidades humanas y del entorno, tienen en la vida diaria; es un factor preponderante para que quienes habitan este mundo moderno califiquen su participación e integración al mismo como sinérgica. Sin embargo, los avances tecnológicos y científicos que emanan del progreso de dicha sociedad, nutren diversos aspectos cotidianos como lo son, la salud y la calidad de vida. Es por ello, que a nivel mundial, la industria biomédica muestra una tendencia de alto crecimiento, la cual, atiende no solo a las demandas del sector salud; sino, que los éxitos en términos de atención médica, respondan al incremento de la expectativa de vida y el acrecentamiento de enfermedades crónico-degenerativas de la población.

Hoy en día, los avances en implantología, han facultado a los investigadores para conocer las reacciones que ocurren entre los tejidos y los diversos implantes. Lo anterior lleva a que se reexamine continuamente sobre el diseño y formulación de nuevos materiales con la objetivo de llegar al implante que, además de ser no tóxico, resistente a la fatiga y a la corrosión, promueva la neoformación de tejido con adhesión celular sobre su superficie; siendo esto último, un paso crucial para que el tejido se integre estructural y funcionalmente al biomaterial. Por ejemplo, la hidroxiapatita (HA), una biocerámica con fórmula química básica $\mathrm{Ca}_{10}\left(\mathrm{PO}_{4}\right)_{6}(\mathrm{OH})_{2}$, se ha empleado como biomaterial o componente de biomateriales compuestos, destinados a reparaciones o sustituciones óseas, debido a sus excelentes características de biocompatibilidad, no toxicidad, estabilidad química, osteoconducción y bioactividad [I-IV].

Esta apatita, que forma parte de la familia de los fosfatos de calcio, puede obtenerse a partir de la transformación de materiales naturales como los esqueletos minerales de los vertebrados, o puede ser sintetizada artificialmente [V,VI]. Dependiendo del origen y del método de síntesis, resultarán materiales con gran variabilidad fisicoquímica y morfológica. Por lo cual, pueden obtenerse distintas hidroxiapatitas que satisfagan los requisitos de aplicaciones clínicas específicas [VII, VIII].
Cabe señalar que las ventajas del método de síntesis por combustión en solución (SCS) sobre otras técnicas químicas, radican en, la utilización de la energía interna del sistema, en lugar de fuentes externas, y, la rapidez en la que se lleva a cabo el proceso; esto debido al calentamiento progresivo del frente de combustión [IX-XI]. Aprovechando estos elevados gradientes térmicos, es posible obtener productos con favorables condiciones en cuanto a tamaño de partícula, siempre y cuando se logre el control requerido en los parámetros relativos del sistema, tales como reactivos precursores, agente combustionante, temperaturas y tiempos de reacción [XII-XIV].

El presente trabajo, tiene como objetivo, determinar la ruta crítica a seguir para realizar la síntesis de polvos de HA de orden nanométrico, mediante el método químico de combustión en solución. Los parámetros de importancia a estudiar para la optimización de la técnica son: fuentes de calcio y fosfato, tipo de combustible, estequiometría, solubilidad de las especies y temperatura de combustión. El material obtenido tendrá un aspecto de polvo fino, mismo que puede ser procesado a futuro para conformar substratos tipo blanco requeridos por ejemplo en la técnica física de crecimiento de películas delgadas conocida como erosión iónica tipo magnetrón con radiofrecuencia [XV].

Lo anterior, de acuerdo con reportes en la literatura, en los que refieren, que las propiedades con las que cuenta la HA, permiten realizar tanto el diseño de materiales de uso biomédico como las predicción de su comportamiento fisicoquímico, $\mathrm{y}$, de esa manera estimar su función y posible aplicación en sistemas reales.

\section{Materiales y métodos}

\section{Descripción metodológica}

El procedimiento a desarrollar, consiste de dos fases que describen el método de combustión química en solución, en el cual, es necesaria la presencia de un agente combustible que detone la reacción dentro del sistema; cuyo calor liberado se traduce en la energía conformacional del compuesto estequiométrico. Inicialmente se obtiene un desecado precalentado y posteriormente vía una reacción exotérmica de alta temperatura, se logra el producto deseado en forma de polvo [XII,XIII, XVI]. 


\section{Preparación de la muestra}

Las materias primas utilizadas para la síntesis fueron, $\mathrm{Ca}\left(\mathrm{NO}_{3}\right)_{2} * 4 \mathrm{H}_{2} \mathrm{O}$ y $\left(\mathrm{NH}_{4}\right)_{2} * \mathrm{HPO}_{4}$ como fuentes de $\mathrm{Ca}$ y $\mathrm{P}, \mathrm{CH}_{6} \mathrm{~N}_{4} \mathrm{O}$ y $\mathrm{C}_{2} \mathrm{H}_{5} \mathrm{NO}_{2}$ como agentes combustibles; $\mathrm{HNO}_{3}$ como exceso de oxidante para garantizar la plena reacción del combustible y controlar el pH de la solución. Las cantidades empleadas fueron calculadas para obtener $1 \mathrm{~g}$ de producto final, considerando la reacción estequiométrica. En la figura 1 se muestra el diagrama de flujo de la ruta de síntesis inicial, la cual se basa en la formación en primera instancia de un producto de apariencia viscosa (desecado) a baja temperatura, seguido de la quema abrupta del mismo por exposición a alta temperatura (combustión).

Primeramente, 0.01 moles y 0.006 moles respectivamente de los reactivos fuente de calcio y fosfato fueron disueltos en $25 \mathrm{ml}$ de agua desionizada. Posteriormente y para dos experimentos por separados, 0.008 moles de carbohidrazida y 0.007 moles de glicina respectivamente fueron disueltos en $25 \mathrm{ml}$ de agua desionizada y adicionados a la solución de reacción, mantenida en agitación constante durante 40 minutos a $80^{\circ} \mathrm{C}$ de temperatura. Finalmente, la mezcla obtenida fue colocada en un horno precalentado a $550^{\circ} \mathrm{C}$. La muestra final fue tratada térmicamente a $700^{\circ} \mathrm{C}$ y $800^{\circ} \mathrm{C}$ respectivamente durante $1 \mathrm{~h}$. y $2 \mathrm{~h}$.

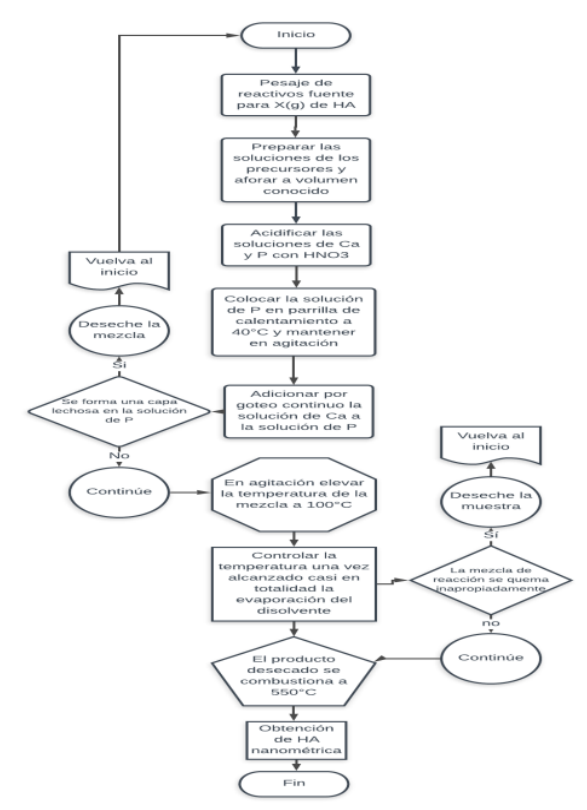

Figura 1 Diagrama de flujo de la ruta de síntesis

\section{Caracterización de la muestra}

Las estructuras cristalinas de los especímenes obtenidos fueron descritas por microscopía de difracción de rayos X (DRX- equipo Phillips $\mathrm{X}$ 'Pert). Los difractogramas correspondientes a cada polvo, se procesaron mediante un programa de análisis, para identificar las fases cristalinas presentes.

\section{Resultados y discusión}

\section{Desarrollo del método}

La metodología planificada permite la obtención del producto de interés, sin embargo la técnica requiere un mejor control de la temperatura de trabajo en la primera fase para evitar que el desecado inicie la ignición a destiempo y en un ambiente con distribución de energía térmica no uniforme. Desde el punto de vista estequiométrico, la quema prematura sugiere la presencia de fases cristalinas no deseadas.

\section{Ruta de síntesis}

De acuerdo a los resultados preliminares, es necesario hacer modificaciones en la ruta de síntesis para establecer ventajas y desventajas en cada una de las partes del protocolo experimental. Lo anterior, permite determinar los ajustes en las variables de interés del sistema, para la debida corroboración de los mismos en corridas experimentales subsecuentes. De aquí, que se podrán establecer las condiciones adecuadas y los parámetros óptimos para el desarrollo de la técnica, y, se garantice la calidad del polvo obtenido.

\section{Análisis de los patrones de difracción}

Los polvos obtenidos para cada ruta con los dos tipos de combustibles, develan en ambos casos la presencia de más de una fase cristalina. La fase primaria es, HA (código de referencia no. 09-0432) y la fase secundaria es $\beta$-fosfato tricálcico ( $\beta$-FTC), (código de referencia no. 09169); las cuales prevalecen aún después del tratamiento térmico. La figura 2, muestra el comparativo de los difractogramas para las muestras tratadas a $800^{\circ} \mathrm{C}$ por $2 \mathrm{~h}$, en la cual, no es posible distinguir diferencia alguna; lo cual sugiere que presentan estructuras semejantes que pudieran ser concordantes con HA pero no en su forma pura. 
La figura 3, muestra también el comparativo entre patrones correspondientes a ambas muestras, pero tratadas a $700^{\circ} \mathrm{C}$ por $1 \mathrm{~h}$. La tendencia de deslizamiento en el difractograma para carbohidrazida sugiere la presencia de una fase secundaria que pudiera ser $\beta$-FTC, o fostato octacálcico; sin embargo, el patrón de Glicina presenta mejor concordancia con los picos representativos de HA cristalina.

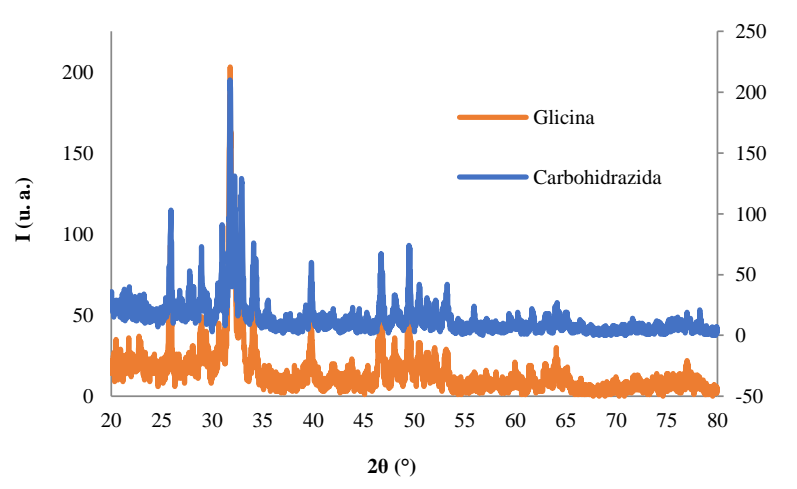

Figura 2 Difractograma de HA tratada a $800^{\circ} \mathrm{C}$ durante $2 \mathrm{~h}$

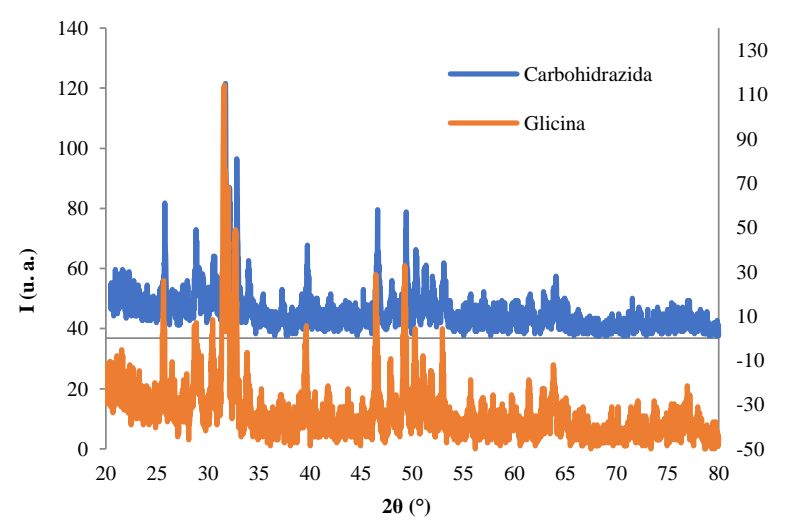

Figura 3 Difractograma de $\mathrm{HA}$ tratada a $700^{\circ} \mathrm{C}$ durante $1 \mathrm{~h}$

\section{Agradecimiento}

Los autores agradecen a la Universidad Autónoma de Baja California por el apoyo concedido a través de la 20 Convocatoria Interna de Proyectos de Investigación.

\section{Conclusiones}

Se obtuvieron polvos de HA mediante la ruta de síntesis diseñada, mismos que poseen la estequiometría propuesta; sin embargo los hallazgos en los patrones de difracción de las muestras, sugieren modificaciones en la metodología a fin de perfeccionar la técnica y lograr obtener un producto con las características estructurales deseadas.
En relación a la contribución que el agente combustionante tiene en la conformación del compuesto, se concluye que el más eficaz, dentro de los rangos de temperaturas y tiempos de trabajo, es la glicina. Para fines de una apropiada caracterización, deberán realizarse, una serie de experimentos, que den seguimiento tanto a la optimización de parámetros, como al análisis microscópico correspondiente.

\section{Referencias}

I. Elliot J.C., Wilson R. M., Dowker S.E. (2002). Apatite Structures. Adv. X-ray Analysis, 45, 17281.

II. Dorozhkin S. (2009). Calcium Orthophosphates in Nature, Biology and Medicine. Materials, 2, 399-498.

III. Ghosh S.K., Nandi S. K., Kundu B., Datta S., De D. K, Roy S. K., Basu D. (2008). In vivo response of porous hydroxyapatite and $\beta$ tricalcium phosphate prepared by aqueous solution combustión method and comparison with bioglass scaffolds. J. Biomed. Mater Res. Part B: Applied Biomaterials, 86B, 217-27.

IV. Lopes J., Vargas M. O., Odone I., Aparecido C. (2019). Investigation of citric acid-assisted sol-gel synthesis coupled to the self-propagating combustion method for preparing bioactive glass with high structural homogeneity. Materials Science and Engineering C, 97, 669-78.

V. Türk S., Altinsoy İ., CelebiEfe G., Ipek M., Özacar M., Bindal C. (2017). Microwave-assited biomimetic synthesis of hydroxyapatite using different sources of calcium. Materials Science and Engineering $C, 76,528-635$.

VI. Chouddhary R., Manohar P., Vecstaudza J., Yáñez-Gascón M., Pérez S. H, Nachimuthu R., Locs J., Swamiappan S. (2017). Preparation on nanocrystalline forsterite by combustion of different fuels and their comparative in-vitro bioactivity, dissolution behavior and antibacterial studies. Materials Science and Engineering C, 77, 811-22.

VII. Nayak A. K. (2010). Hydroxyapatite synthesis methodologies: an overview. International Journal of ChemTech Research 2 (2), 903-7. 
VIII. Zhao J., Dong X., Bian M., Zhao J., Zhang Y., Sun Y., Chan J., Wang X. (2017). Solution combustion method for synthesis of nanostructured hydroxyapatite, fluorapatite and chlorapatite. Applied Surface Science, 314, 1026-33.

IX. Patil K., Aruna S.T., Mimani T. (2002). Combustion synthesis: an update. Current Opinion in Solid State and Materials Science, 6, 507-12.

X. Varma A., Mukasyan A., Rogachev A., Manukyan K. (2016). Solution Combustion Synthesis of Nanoscale Materials. Chem. Rev., 116, 14493-586.

XI. Wen W., Wu J. (2014). Nanomaterials via solution combustion synthesis: a step nearer to controllability. RSC Adv., 4, 58090-100.

XII. Ayers R., Hannigan N., Vollmer N., Unuvar C. (2011). Synthesis of heterogeneous calcium phosphate bioceramics from calcium oxide and phosphate precursors. International Journal of Self-Propagating High-Temperature Synthesis, 20, 6-14.

XIII. Cüneyt Tas A. (2000). Combustion synthesis of calcium phosphate bioceramic powders. Journal of the European Ceramic Society, 20, 2389-94.

XIV. Aruna S., Mukasyan A. (2008). Combustion synthesis and nanomaterials. Current Opinion in Solid State and Materials Science, 12, 44-50.

XV. Ozeki K., Masuzawa T., Aoki H. (2017). Fabrication of hydroxyapatite thin films on polyetheretherketone substrates using a sputtering technique. Materials Science and Engineering $C, 72,576-82$.

XVI. Krubowski S., Lysenko N., Kolodziejski W. (2018). Synthesis and characterization of nanocrystalline composites containing calcium hydroxyapatite and glycine. Journal of Solid State Chemistry, 264, 59-67. 